\title{
BACTERIOLOGICAL AND CLINICAL OBSERVATIONS ON THE TREATMENT OF THE ACUTE OPHTHALMIAS OF EGYPT WITH SULPHONAMIDES AND PENICILLIN*
}

\author{
BY \\ J. O. W. Bland \\ PATHOLOGIST, MEMORIAL OPHTHALMIC \\ LABORATORY, GIZA, CAIRO, EGYPT \\ and \\ Rowland P. Wilson \\ DIRECTOR, MEMORIAL OPHTHALMIC \\ LABORATORY, GIZA, CAIRO, EGYPT
}

DURING the past decade, chemotherapy with the sulphonamide drugs has been extensively used in ophthalmology and, inasmuch as the acute ophthalmias of Egypt are caused by bacteria which are sulphonamide-sensitive, their use in the treatment of these diseases was particularly indicated.-

A considerable amount of experimental work on this subject has already been carried out at the Memorial Ophthalmic Laboratory since 1937 and papers have been communicated to the Ophthalmological Society of Egypt by Nashed, ${ }^{1} \mathrm{Hilmi} \mathrm{Hamid}^{2}$ and Wilson. ${ }^{3}$

These communications show that so far as sulphanilamide and sulphapyridin are concerned, sulphapyridin is much more effective in the treatment of the acute ophthalmias than sulphanilamide; especially does this apply in the case of acute conjunctivitis due to the Koch-Weeks bacillus upon which sulphanilamide has little or no effect. Sulphapyridin has proved therefore to be a most important and valuable weapon in our therapeutic armamentarium, for without question its effect in some cases of gonococcal ophthalmia is little short of miraculous. Furthermore, as a therapeutic agent for both forms of acute ophthalmia; it greatly surpasses any hitherto at our disposal. It can be used entirely unaided by silver nitrate or any other such antiseptic, and indeed it is doubtful if such antiseptics assist its action. Sulphapyridin, therefore, held pride of place for some years although it is not without toxic effects and is inclined to create a certain amount of general malaise and digestive disturbance. However, these are never serious as it is seldom necessary to prolong treatment for more than three or four days.

Among the, newer sulphonamides, sulphathiazole and sulphadiazine have been found to be quite as efficacious as sulphapyridin, and possibly more so, without being as toxic, so it is natural that 
they have tended rather to supplant their more toxic predecessor. Of these two, sulphadiazine is even less toxic than sulphathiazole so that, provided it gives the same excellent results as sulphathiazole, it is likely to be the drug of choice. Experiments were, therefore, devised to test this out so far as their action in the acute ophthalmias is concerned.

' It is usually stated that, in order that the above-mentioned súlphonamide compounds should be effective in the diseases for which they are administered, a standard concentration in the blood of 5 to $10 \mathrm{mgm}$. per hundred cubic centimetres should be maintained, and that, in order to ensure this, 0.5 gram for adults every 2 hours is advised. Emphasis is placed upon the necessity of maintaining an adequate concentration of the drug in the blood. In the following experiments, however, this has not been considered. The criterion has been the bacteriological state of the conjunctiva-in other words we have concentrated our attention on the bacteriological effects of the drug rather than on any particular blood levels. This mode of approach to the subject has not been cominonly studied, for indeed -in many diseases it is impossible to use it, but in infective diseases of the conjunctiva we are in the fortunate position of being able to follow exactly from hour to hour how the drug employed is affecting the conjunctival flora. This is what has actually been done and we have found, to our surprise, that the bacteriostatic effect of both the sulphonamides and of penicillin persists for long past the time when these drugs are ordinarily considered to have fallen below their effective level in the blood. We now propose to outline the results of these experiments. These were designed to elucidate 3 principal points :- -

1. The effect of various sulphonamides upon the pathogenic flora in the acute ophthalmias, i.e., the Koch-Weeks bacillus and the gonococcus.

2. The simplest and most satisfactory method of curing these conditions and the most effective sulphonamide for the purpose.

3 . The relative values of sulphonamides and penicillin in the treatment of these diseases.

\section{'Technique}

Before presenting the results of these experiments, it is important to make a few explanatory remarks regarding the procedure followed. In the first place, the work was carried out on ordinary hospital patients. - Cases of acute purulent or muco-purulent conjunctivitis are not ordinatily admitted to hospital but, for the purposes of these experiments, it was imperative that all cases should be hospitalized, otherwise much exacting labour would have been wasted and carefully timed observations could never have been carried oyt. The method of calculation of dosage 
remained the same throughout the entire set of experiments, and for all children and adolescents was based on a dose of 0.5 gram per 10 kilograms of bodyweight in the 24 hours. As a result of information gained from previous experiments, and in order to reduce treatment to its utmost simplicity and practicability, only two doses were given in the 24 hours. Each dose therefore was a half of the calculated 24 hourly dose. Lest it might be thought that this single dose is dangerously large, it may be noted that it is practically the same as the usual 6-hourly dose designed to give a concentration of $5-10 \mathrm{mgm}$. per $100 \mathrm{c.c}$. of blood. In other words, the total 24-hourly dosage which we employed is actually only a half of that usually recommended.

The calculation of dosage according to bodyweight is, however, not always convenient or practical, so that in actual practice we would suggest one based on age, the following figures serving as a guide :-

\begin{tabular}{|c|c|c|c|c|c|}
\hline $0-3$ months & $\frac{1}{4}$ & ablet ( $\frac{1}{2}$ gram & tablet) & twice & daily \\
\hline $3-6 \quad$, & $\frac{1}{3}$ & , & 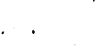 & ", & , \\
\hline $6-12$, & $2 / 5$ & , & - & , & 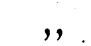 \\
\hline 1 year & $\frac{1}{2}$ & ", & & ;, & $"$ \\
\hline 3 years & $\frac{3}{4}$ & ,, & - & ", & ", \\
\hline ," & 1 & ", & &, & $"$ \\
\hline ", & 1.5 & ," & . & ", & , \\
\hline,, & 2.5 & ,' & & ,' & ," \\
\hline
\end{tabular}

(Tobgy ${ }^{10}$ bases his dosage on 0.5 gram per 20 kilos of bodyweight and states that he has perfect results.)

In Egypt, the great majority of cases occur in children under 10 years of age so that it is seldom necessary to give large amounts at any one time. In adolescents, the 24-hourly dose may preferably be divided into three. In infants, the administration of the drug frequently presents difficulty as part of the dose may be lost through spluttering. One cannot place fragments of a tablet in the mouth of an infant, nor is it satisfactory to put the dry powder into the mouth because this often causes irritation of the pharynx and coughing. It was ultimately found, after trying different methods, that the best way to give the drug was to suspend the dose in 2-3 c.cs. of milk and drop it into the child's mouth with an eye dropper. An alternative way is to make the dose up into a paste with milk or water and to insert it into the child's mouth on the end of one's finger.

- In order to follow the bacteriological progress of the case, smears from the conjunctival discharge were examined before treatment was commenced, and thereafter at regular intervals as the experiment proceeded. At first, both cuttures and smears were made but it was soon found that all essential information 
could be got from the smear alone. An estimate by visual means was 'made of the relative number of organisms in the smear and the result recorded by a system of + signs. The following-points were found important in technique if a just evaluation of numbers is to be attained.

1. Films must not be too thick or too thin, and must be spread evenly so that pus cells cover the field -without clumps.

2. Immediately after taking the films, the everted lids and lid margins are swabbed to remove excess secretion so that the next film shall be made from freshly formed pus.

3. Films are stained by Gram's methods. A good technique is essential.

4. Films are searched for 5 minutes before being pronounced negative. A longer period would have resulted in more positives, but we were interested in relative rather than absolute results.

5. All films were estimated by one observer, so as to keep subjective factors constant.

The system of notation used in recording results in this paper is as follows :

+ signs indicate gonococci, $\times$ signs Koch-Weeks bacilli.

- = a negative film.

$+=$ Not more than 3 fields containing organisms were found in a 5 -minute search.

$++=$ Organisms in more than 3 fields but at wide intervals.

$+++=$ Organisms not found in every field but found in every few fields.

$+++t=$ One or a few organisms in every field.

$+++++=$ Numerous organisms in every field.

$++++++=$ Every field crowded with organisms.

We are fully aware that this must seem a very rough and ready way of estimating the degree of bacterial multiplication in the conjunctiva and are alive to its inaecuracies, but the uniformity of our results show that the technique was, in fact, adequate for the purpose. The different grades in our stheme of enumeration are sufficiently well marked to be easy to differentiate when examining a film and have some real meaning even though this may seem rather imprecise:

A similar system was used for recording the main clinical signs : swelling and discharge. Thus, the degree of infection and the severity of the clinical state could be recorded at the outset and followed graphically throughout the experiment. 


\section{Results}

1. Description of Experiments and Bacteriological Effects. Experiment No. $1-A$ single dose of sulphapyridin. (Table I). -A group of 7 children showing heavy infections, of which one was pure gonococcus, one a pure Koch-Weeks and 5 were mixed when seen in the morning. They were given a dose of sulphapyridin by mouth at 11 p.m. by which time the bacterial state of their conjunctivae was little changed. Hourly observations were begun 8 hours later.

This experiment showed that, except for the pure K.W. infection which proved extremely resistant, reduction of the numbers of bacteria must have begun during the 8 hour period when no observations were taken and was complete between 8 and 12 hours after administration of the drug. All but the pure K.W. case remained negative up to at least 21 hours after administration of the drug, three were still negative after 3 days and were considered cured. Two originally mixed cases showed a relapse on the 2 nd day, one showing a heavy K.W. infection and the other.. a doubtful gonococcal infection which disappeared the next day. This case also can be considered a cure. One further case relapsed with gonococcal infection on the 3 rd day. The resistant .K.W. infection required three further doses of the drug, two on the 1st day following the original dose and one on the 2nd before it was cleared of bacilli.

This experiment shows the remarkable value and possibilities of this drug, a single dose of which cured nearly 60 per cent. of the cases. Such a result was quite unexpected. We had not expected a single dose to cure any case.

EXPERIMENT NO. 2-The effect of 2 doses of sulphapyridin on 1 day. (Table II).-The previous experiment suggested that a single dose probably begins to be effective about 5 hours after administration and remains so for at least 24 hours. If a second dose is to be given, it should be timed to become effective within this period, and therefore should not be given later than 19 hours after the first. In practice, this would be an awkwardly long interval, and in the following experiment we chose one of 9 hours. A group of 9 children showing heavy infections, 1 pure gonococcal, 3 pure Koch-Weeks, 5 mixed, were given a dose of sulphapyridin and a second 9 hours later. Conjunctival smears examined hourly to the 12 th hour showed that the drug begins to be effective, as supposed, about the 5th hour and that clearing is complete, or almost so, by the, 12 th. All the cases were negative 21 hours after the 1 st dose and 5 were completely cured including 1 pure gonococcus and 1 pure $\mathrm{K} . \mathrm{W}$. infection. The remaining 4 cases showed relapses on the 3 rd or 4 th day of the experiment and required further treatment. 


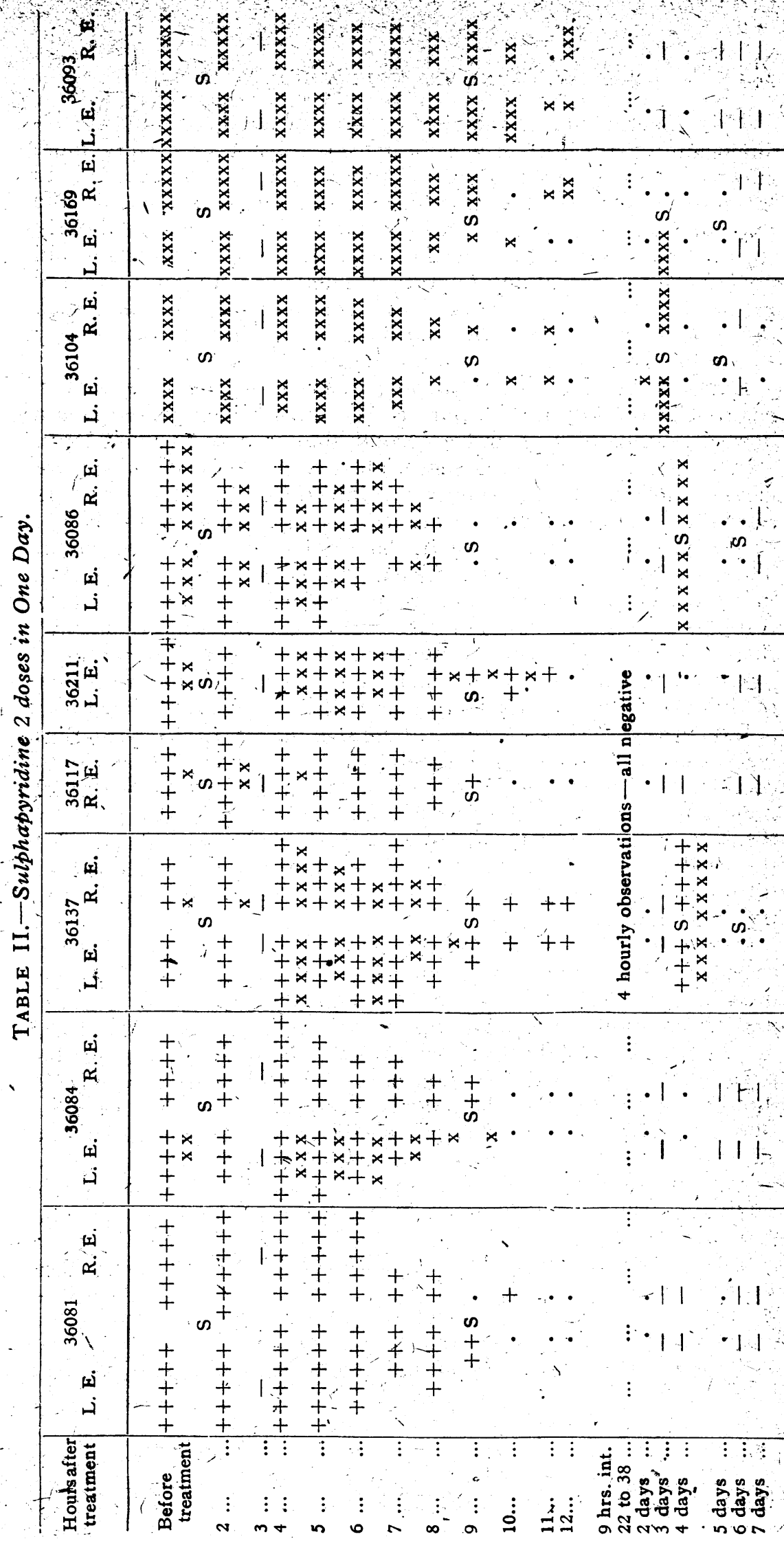


This experiment showed that 2 doses of sulphapyridin are insufficient to cure all cases and indeed are not noticèably more effective than a single dose. Consequently, we proceeded to examine the effects of sulphathiazole and sulphadiazine. In the experiments with these drugs, observations were made 4 hourly for the first two days instead of hourly, since these intervals would be sufficient to reveal any important difference between the rate of action of these drugs and that of sulphapyridin. No such difference was found to exist and therefore it will not be necessary to exhibit these experiments in the form of charts.

Experiment No. 3-2 doses of sulphathiazole on 1 day.-Ten cases of heavy infection, 3 pure K.W. and 7 mixed, were given a dose of sulphathiazole and a second similar dose 8 hours later. Twelve hours after the first dose, all except one K.W. case were clear of bacteria or showed only a + infection. Twenty-four hours after the first dose all were clear. Seven, including 1 of the K.W. cases, remained so up to the 4th day and were discharged cured. Three cases, $2 \mathrm{~K}$.W. and 1 mixed, showed recurrences of K.W. infection on the 3rd day and required further treatment. This result suggests that sulphathiazole may be a little more effective than sulphapyridin, but the number of cases in the two series is too small to warrant a firm conclusion. In any event, two doses of this drug are not sufficient to cure all cases of K.W. infection.

EXPERIMENT NO. 4-4. doses of sulphathiazole in 2 days.-Sixteen cases of heavy or moderately. heavy infection, $\tau$ pure gonococcal and 9 mixed, were given 4 doses of sulphathiazole; at 12 noon and $10 \mathrm{p} . \mathrm{m}$. on the first day and at $10 \mathrm{a} . \mathrm{m}$. and $7 \mathrm{p} . \mathrm{m}$. on the second day. Observations were made daily for 5 days. All the cases were clear of bacteria after 24 hours and remained so throughout. This scheme of treatment therefore resulted in 100 per cent. cures.

EXPERIMENT No. 5-2 doses of sulphadiazine on 1 day.-Twelve cases of heavy infection, 7 pure gonócoccal, 1 pure K.W., 4 mixed, were given 2 doses of sulphadiazine at 8 hours interval and observations were made up to the 5 th day. All were clear, or almost clear, of bacteria by the 12 th hour. All, except the pure K.W. case and one mixed case, were completely cured, though two of them could only be followed up to the 3rd day. Both the relapses were of K.W. bacilli only. This result shows an improvement on the results of similar treatment with sulphapyridin or sulphathiazole, but again the number of cases is too small for firm conclusions and in any case the method does not cure all cases.

EXPERIMENT No. 6-4 doses of sulphadiazine in 2 days.-Ten cases, 1 pure gonococcal and 9 pure K.W. infections, were given 2 doses of sulphadiazine on each of two successive days and were followed with daily observations for 5 days. All were clear of bacteria 24 hours after beginning treatment and all were cured. 
TREATMENT OF ACUTE OPHTHAMLAS OF EGYPT WITH SULPHONAMIDES ANG PENICILLIN

A summary of the results of these 6 experiments is given in tabular form in Table III.

TABLE III-Results of Six Experiments-with Sulphonamides.

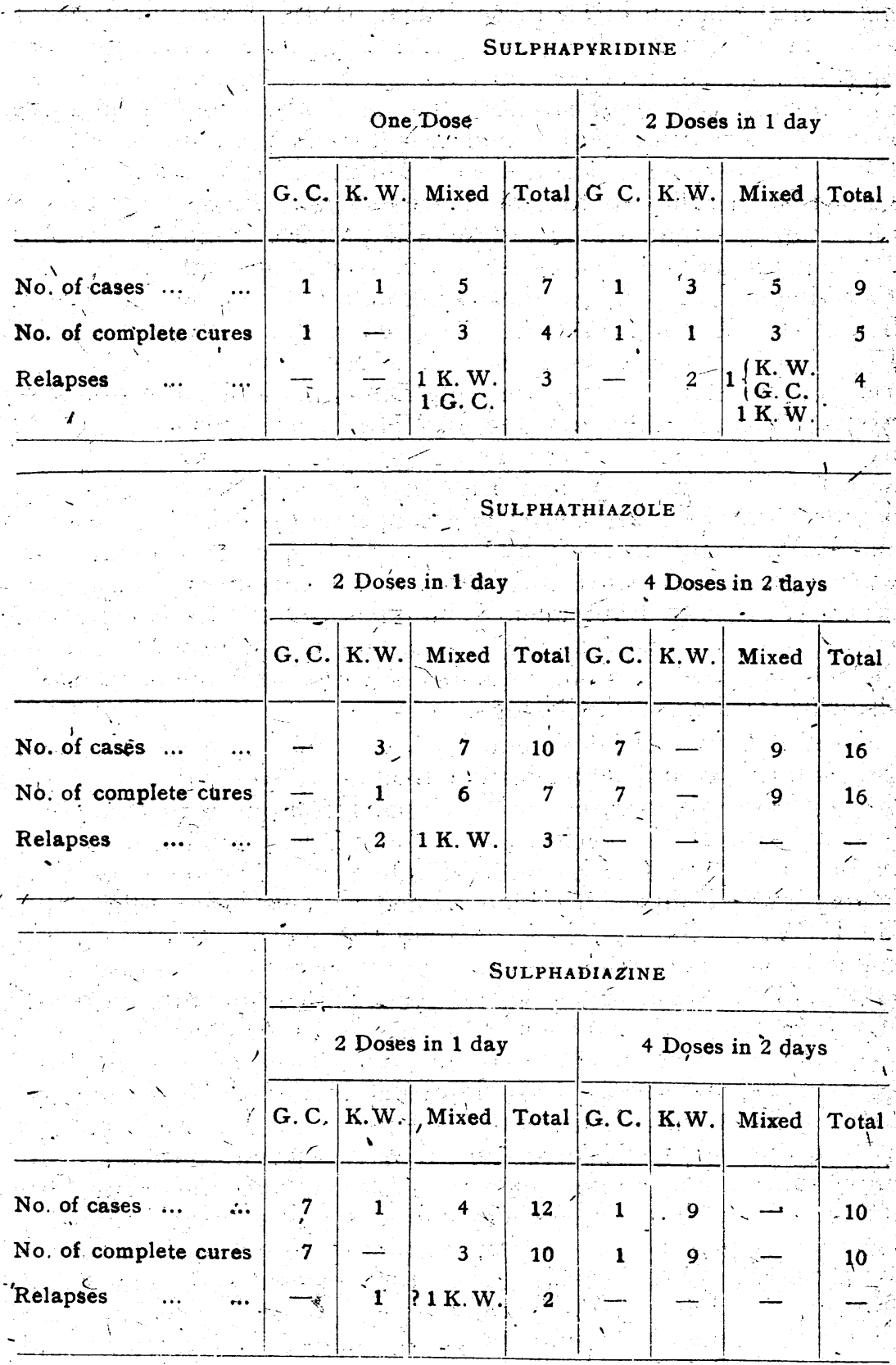


The number of cases used in any one of our experiments is not large and might indeed be thought too small to justify conclusions, but certain features of the results in all the experiments have proved constant and reveal that there is a close similarity between the action of all these drugs. Thus, Experiment No. 1 and Experiment No. 2 taken together suggest that a single dose of sulphapyridin produces no effect on the conjunctival flora up to 4 hours after administration, that soon after the 4 th hour the action of the drug begins and that this is pronounced after 8 hours. Experiments 3 and 4 with 2 doses of sulphathiazole and sulphadiazine showed the same phenomena. Experiment No. 1 also suggests that a single dose will produce complete clearing of the conjunctiva in most cases at, or shortly after, the 12th hour. Experiment No. 2 shows that administration of a second dose 9 hours later does not influence the speed of this final result; while Experiments 3 and 4 are so similar to Experimen' 2 that we can assume the same is true for sulphathiazole and sulphadiazine. Sixty-four cases,-therefore, the total of all our experiments, make it clear that a single dose of any one of these sulphonamides will be followed by a lag period of about 4 hours before bacteriostasis begins and will cause complete disappearance of both gonococci and $\mathrm{K} . \mathrm{W}$. bacilli in about 12 hours. Only very occasional cases will be found resistant to this action. We encountered only one, the $\mathrm{K} . \mathrm{W}$. case in Experiment 1, in our series of 64. Experiment 1 further shows that a single dose of sulphapyridin will result in the complete cure of a large number of cases, and this is probably also true for the other two drugs.

Turning now to Experiments 2, 3, 4, 5 and 6, designed to 'find the simplest completely effective treatment, we find that if we consider the mixed cases as individual infections with the gonococcus or the K.W. bacillus, then 2 doses were sufficient to cure 22 out of 23 cases of gonococcal infection. The one case that relapsed was a mixed infection treated with sulphapyridin. But the K.W. bacillus is more resistant. None of the three compounds completely eradicates it from all cases with 2 .doses; with sulphapyridin 4 out of 8 cases relapsed, with sulphathiazole 3 out of, 10 and with sulphadiazine 2 out of 5 . Yet it must be emphasized that in every case the conjunctival dischatge was bacteriologically negative for at least the whole of the day following the 2 doses, including the cases that relapsed, and that with sulphapyridin the relapses occurred on the 2 nd, 3rd and 4th days, with sulphathiazole on the 2nd and 3rd, with sulphadiazine on the 4th and 6th: The latter case indeed should probably be counted a fresh infection. Thus it is clear that 2 doses only of any of these compounds might be sufficient to cure practically all gonococcal infections but that further treatment is necessary to ensure that relapses will not occur amongst K.W. cases. Experiment 5 and 
6 show that when 2 further doses of sulphathiazole or sulphadiazine are given on the following day all cases are cured, the numbers concerned being 26,16 with sulphathiazole, 10 with sulphadiazine. Further, although 18 patients had K.W. infection, not one of these relapsed. Either compound may therefore be used with confidence, but the preference must be given to sulphadiazine which is less toxic than sulphathiazole and more rapidly, acting. It is for this reason that we made no four-dose experiments with sulphapyridin for this drug is the most toxic, and judging from our experiments, the least effective of the three.

\section{Clinical effects}

At this stage in the history of sulphonamide treatment, it is unnecessary to discuss in detail the purely clinical effects of these drugs. Everyone now knows how remarkably effective they are in the treatment of ophthalmia. In the clinical signs, however, there is always a time lag behind the bacteriological findings, for although the smears may be negative in 9 hours it takes from 24 hours to 3 days to complete the clinical cure. This delay has undoubtedly influenced us in the past to carry on treatment for 3-4 days-in fact to over-treat our cases. Experiments 5 and 6 make it clear that this is unnecessary. Only if a case shows a bacteriological relapse should further treatment be given. Symptoms are apt to persist longest when the case is complicated by corneal ulceration, but in these cases the discharge no longer contains the original causal organism but only secondary invaders, such as staphylococci or diphtheroids.

\section{Morphological effects on the causal organisms}

We have made no detailed study of this subject but have, nevertheless, made a few observations in the course of the work which may be of interest to others. In the gonococcus, the effects are uninteresting. About the time when the drug begins to become effective, one starts to find large swollen coccoid elements in the smears which are similar to the involution forms found in old cultures of gonococci. These are thereafter regularly seen until the case becomes negative. They are presumably dead cocci.

In the case of $\mathrm{K} . \mathrm{W}$. bacilli, the effects are more interesting. Again, about the time the drug begins its action one notices that the bacilli tend to be longer than normal, and when the drug becomes fully effective, i.e., when the smear reaches the ++ or + stage, this elongation is often pronounced. Single bacilli and small clumps are found which stretch across the whole diameter of the leuicocyte that has engulfed them and sometimes quite long curled filaments are seen. This elongation is not accompanied by any swelling or fattening of the bacilli. This metamorphosis 
may perhaps be related to a similar occurrence we have often noted in culture. When the K.W. bacillus is sown on blood agar, it is usual for it to grow in the form of long bacilli and very long twisted, curled and tangled skeins of filaments. If such a culture is now subcultured on to heated blood agar, in many cases the organism reverts to the regular rod-shaped form of medium length which is its habitual appearance in the ocular discharge. It may be that the elongation of the bacilli in both cases is a result of life in unfavourable circumstances and is related to a disturbance of nutrition, caused in the case of sulphonamides by an interference with metabolism and in culture by an insufficiency in the blood agar of some metabolite supplied by the heated medium. Such a suggestion is, of course, entirely speculative.

\section{Relative'values of penicillin and sulphonamides}

The number of cases treated with penicillin was small, and the few experiments were early abandoned because it rapidly became apparent that penicillin is quite unsuitable for the treatment of the acute ophthalmias whether administered intramuscularly or employed in the form of drops. The following is a brief summary of the results obtained by intramuscular injections, and Tables IV and $\mathrm{V}$ are typical illustrative charts.

EXPERIMENT NO. 1.-Penicillin 4 hourly for 1 day (360 units per kilo of bodyweight per dose). 3 cases G.C. ophthalmia-2 cases recurred.

EXPERIMENT NO. 2.-Penicillin 3 hourly for the first 24 hours (275 units per kilo per dose).

4 hourly for the second 24 hours (360 units per kilo per dose).

One case G.C. ophthalmia-G.C. cured-no recurrence, but became infected with K.W. which was unaffected by penicillin.

One case K.W. ophthalmia-no effect.

Both K.W. infections yielded satisfactorily to sulphapyridin:

EXPERIMENT NO. 3.-Penicillin-2 injections at 24 hourly intervals (360 units per kilo of bodyweight per dose).

7 cases $\left\{\begin{array}{l}3 \text { G.C. } \\ 4 \text { mixed K.W. and G.C. }\end{array}\right\}$ No cure of either type.

EXPERIMENT NO. 4.-Penicillin at 12 hourly intervals for 2 days (730 units per kilo per dose).

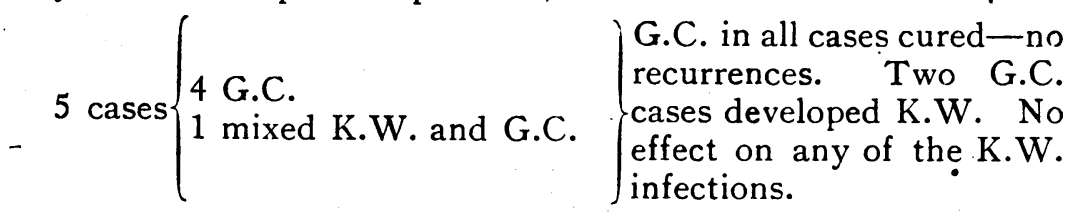

The results obtained when employing topical application were even less encouraging. Every precaution was taken to ensure 
that only highly active solutions of calcium penicillin were used but only one concentration was employed, viz., 500 Oxford units per c.c. Drops were instilled at approximately hourly intervals during the day only, it being impossible to arrange for treatment during the night. In addition the eyes were washed out with saline each morning (and if necessary at other times of the day when discharge was copious). The following results were obtained :-

(1) Gonocóccal Ophthalmia.-Eleven cases in all were treated, of which 8 were cured in an average of 6 days. In 3 cases the response was so unsatisfactory that, fearing complications, sulphonamides were resorted to on the 2 nd, 3rd and 8 th days respectively. In 2 cases, $\mathrm{K}$.W. bacilli appeared in the discharge during treatment and for these also sulphonamides had to be employed to complete the cure.

(2) Koch-Weeks Ophthalmia.-Of 8 cases treated, only one was completely cured and that only after 8 days. Two cases were unaffected by treatment and actually became worse, so that sulphonamides had to be resorted to. In 5 cases, there was an apparent cure, but the conjunctival sac in each case still contained K.W. bacilli at the end of treatment. Two of these cases relapsed several days later.

Actually, it is doubtful if the penicillin had any therapeutic value in any of these cases, for the improvement was probably due to the saline washouts and to natural healing processes. The average time taken from onset of disease to final "clinical", cure was 10 days.

Conditions and known facts did not encourage us to test out the effect of stronger concentrations of penicillin, for Garrod ${ }^{4}$ has shown that nothing is likely to be gained by using high concentrations particularly for local treatment.

The results of our experiments permit the following conclusions :

Penicillin given intramuscularly is highly potent in gonococcal ophthalmia (Table IV). A single injection brings about a complete disappearance of gonococci from the conjunctival discharge within 2-3 hours and will maintain the eye free of gonococci for about 10 hours, a rather surprisingly long period, but repeated injections over at least 2 days are necessary to obtain a complete cure and, moreover, further experiments by Sabri Kamel ${ }^{5}$ have shown that relapses may occur if 12 hourly injections are employed (cf. Experiment 4). Topical applications are extremely laborious and would impose an enormous strain on the nursing staff if (as ought to be done) drops are employed both day and night. The results in no way justify such a method of treatment seeing it has been proved that the sulphonamides are so eminently effective and satisfactory. Furthermore, penicillin utterly fails to 


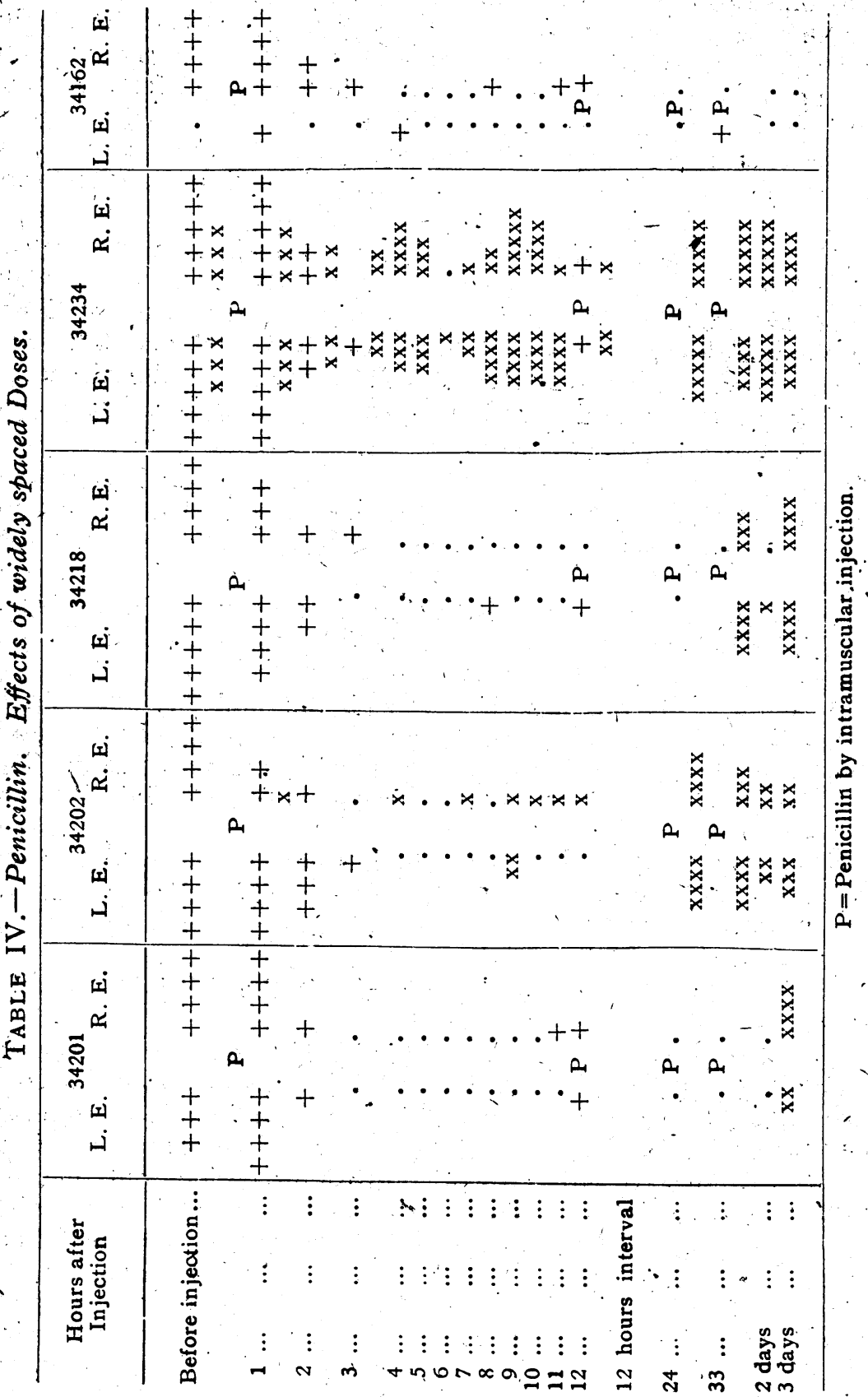




\section{Treatment of ACUTE Ophthalmias of' EgyPt With SUlPHONAMIDES AND PENICILLIN}

reduce in any way the number of bacilli in $\mathrm{K}$.W. cases even when given 3-hourly by injection, indeed the bacilli actually appear to increase in numbers despite the fact that such cases may show some slight clinical improvement (Table $\mathrm{V}$ ), a result we fully expected considering the close relationship, if not identity, of the K.W. bacillus and $H$. influenzae which is not sensitive to penicillin. Penicillin is therefore of no value whatsoever in $\mathrm{K} . \mathbf{W}$. conjunctivitis whether by injection or locally, so that in- at least 50 per cent. of the cases of ophthalmia in Egypt it is not only useless but is contraindicated as it is very likely to leave the case

TABLE V.-Penicillin. A Koch-Weeks case treated with 3 hourly injections. (Case No. 31157).

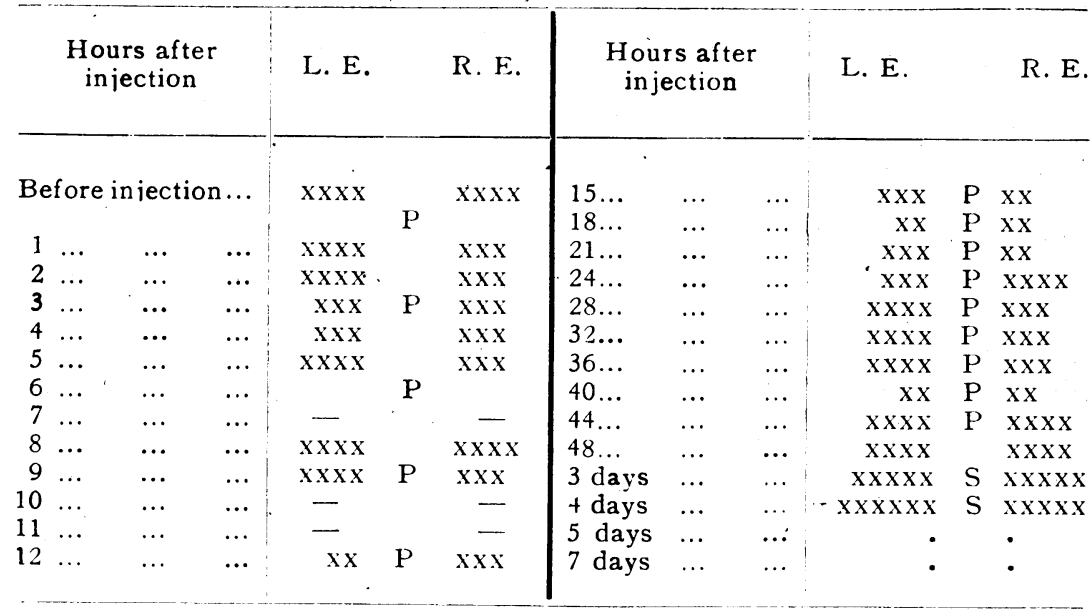

$\mathrm{P}=$ Penicillin by intramuscular injections.

$\mathrm{S}=$ Sulphapyridin 2 doses daily.

a carrier. Even in pure gonococcal ophthalmia, penicillin parenterally has no real advantage over sulphathiazole or sulphadiazine, for although the sulphonamides take 6-9 hours longer to render the conjunctival discharge bacteriologically negative, this initial delay is of little consequence. It is unlikely that by the slightly more rapid action of penicillin any serious complications, such as perforation and prolapse of iris, would be averted. In the purely gonococcal case, the speediest cure would be most surely obtained by repeated injections of penicillin, but this demands treatment which can only be given in hospital and even then is difficult to apply in Government Ophthalmic Hospitals in Egypt (where cases occur by the hundreds of thousands), and also demands that the diagnosis should be carefully established by smear examination beforehand as it is useless to persist with penicillin either in pure K.W. cases or in mixed cases.

On the other hand, the treatment with sulphonamides which 
we have suggested could not possibly be more simple or more easily carried out, does not require hespitalization and is alike successful in both gonococcal and Koch-Weeks ophthalmia.

\section{Discussion}

Those who know the conditions of Egyptian life ${ }^{6}$ or have studied the work of Wilson ${ }^{6-9}$ will realise the import of our experiments. Each year, an epidemic of acute ophthalmia afflicts that country 7,9 striking particularly at the infants and small children, of whom not one except among a negligible minority of the very rich escapes an attack before its second year of life.7,8 This scourge is the cause of 80 per cent. of the blindness (total blindness 1 per cent., monocular 4 per eent.) of E'gypt and leaves behind, in addition, a multitude of more or less serious ocular defects. ${ }^{6}$ The increased ocular secretion caused by the disease promotes also the spread of trachoma, ${ }^{8}$ that second universal plague of Egypt, itself a fertile source of damaged sight. No treatment which requires hospitalization or even attendance at hospital, no treatment which cannot be taken to the villages,. where the great majority of the people live, and there simply and easily administered, can hope to do more than scratch the surface of this problem. The treatment with sulphonamides which we have described is exactly such. It is simple, safe, effective and could be given by unqualified persons with a minimum of training ; it provides, we believe, the necessary solution, not only for Egypt but for other oriental countries where similar conditions of life and disease prevail. Made available in the villages, it would soon be used by the people and would result in a great diminution of the evil effects of ophthalmia. Yet, more important than its value to the afflicted sufferers, it offers the hope of checking and even, perhaps, of eradicating the yearly epidemic. The untreated case of acute ophthalmia discharges living and virulent organisms from its eyes for at least 6 days, during the whole of which period it is a source of infection for its neighbours. By sulphonamide treatment, this period can be reduced to perhaps 1 or 2 days, for any case will be rendered non-infective 12 hours after the first administration of the drug. A reduction of the infective period to $1 / 6$ of the usual will, if most cases are treated, undoubtedly have an effect on the course of the epidemic. How great that effect will be, we cannot tell without a field experiment on a large scale, but it is not unreasonable to hope that it might be decisive. It is of the utmost importance that such field experiments be done and the treatment made, available in all countries where this disease occurs.

\section{Summary and conclusions}

1. The effect of various dosage with sulphapyridin, sulphathiazole, sulphadiazine and penicillin on the bacterial condition of 
the conjunctiva was studied by means of repeated smear examinations in the acute epidemic ophthalmias (Koch-Weeks and gonococcal) of Egypt.

2. A single dose of sulphonamide will cause a reduction of bacteria in four hours and total suppression of bacteria in about 12 hours. A considerable proportion of gonococcal cases will be cured by a single dose but Koch-Weeks cases are more resistant.

3. Two doses at 8-hour intervals in, one day of sulphathiazole or sulphadiazine will cure almost all gonococcal cases but not all Koch-Weeks cases.

4. Two doses on two successive days ( 4 doses in all) will cure all cases of gonococcal and Koch-Weeks ophthalmia with very rare exceptions.

5. A single intramuscular dose of penicillin will reduce gonococci to nil in 3-4 hours but repeated doses at short intervals are necessary to ensure cure. Relapses occur in 10-12 hours after a single dose.

6. Penicillin has no effect on Koch-Weeks cases and is therefore unsuitable as a treatment for acute Egyptian ophthalmia.

\section{REFERENCES}

1. NASHED, E. (1940)-Bull. Soc. Ophthal. Egypt, Vol. XXXIII, p. 64. (1942).-Bull. Soc. Ophthal. Egypt, Vol. XXXV (not yet printed).

2. Hamid, H. (1943).-Bull. Ophthal. Soc. Egypt, Vol. XXXVI (not yet printed).

3. Wilson, R. P. (1938).-Thirteenth Annual Report, Mem Ophthal. Lab., Giza, Cairo, p. 103.

4. Garrod, L. P. (1945). - Brit. Med. Jl., January 27, p. 107.

5. KAMEL, S. (1945).-Personal communication.

6. Wilson, R. P. (1929).-Ann. Rep. Mem. Ophthal .Lab., Giza, Cairo.

7. (1933-36).-Ann. Rep. Mem. Ophthal. Lab., Giza, Cairo.

8. (1936)-Comptes Rendus, International Association for Prevention of Blindness.

9. - (1935).-Bull. Soc. Ophthal. Egypt, Vol. XXVIII.

10. Toвgy, A. F. (1943). -Bull. Ophthal. Soc. Egypt, Vol. XXXVI (not yet printed).

\section{BRANCH THROMBOSIS OF MIDDLE CEREBRAL ARTERY*}

BY

\section{KeNNETH C. WybaR}

(FROM THE TENNENT INSTITUTE OF OPHTHALMOLOGY, WESTERN INFIRMARY, GLASGOW)

A woman, aged 60 years, whilst shopping one day at the beginning of April, 1944, experienced a severe left-sided headache. She noticed that she could only see the left-hand side of the shopping list which she was holding in her hand. These symptoms occurred suddenly and concurrently. In an endeavour to

\footnotetext{
* Received for publication, December 18, 1944.
} 